\title{
NIDCAP Rounds: Reaching More Babies and Families
}

\section{Giesen J', Campbell C ${ }^{2}$}

'Speech Pathology and Audiology, Stollery Children's Hospital, Edmonton, Alberta, Canada

2 Occupational Therapy and Physiotherapy, Stollery Children's Hospital, Edmonton, Alberta, Canada

\section{Aims}

The purpose of this project was to institute developmental care rounds in our NICU. The premise for the project arose when, upon completion of training to become a NIDCAP Professional, this writer posed the question, "Now that we've completed training, how do we reach more babies in our limited time? What does it look like in practice?" The aim was to work together with families to learn how to interpret their babies' communication, to support their babies' positions, touch them, talk to them, and hold them in a way that they are able to attend, respond, and interact socially.

\section{Methods}

The ENTCC (Edmonton NIDCAP Training Centre Canada) and rehabilitation therapists at the Stollery Children's Hospital teamed up to develop a plan for instituting NIDCAP Rounds. These were set to occur for two hours every two weeks from October 6, 2020 to the present, excluding holidays. NIDCAP certified participants would include a speech language pathologist (SLP), occupational therapist (OT), and another NIDCAP certified member (nurse, neonatal nurse practitioner, or neonatologist) when available. Eligibility for family participants included those who were:

a) available for rounds

b)and interested in working with the team to learn more about their babies, how to connect with them, and how to support their development and

c) and expected to require a long stay in the NICU

Input was elicited from head nurses and other NICU team members to select families to approach for NIDCAP rounds. The plan was to visit with three families each round. At the start of each round, one team member would introduce NIDCAP and the premise of rounds, providing a handout to families for reference. Families were encouraged to describe their babies, what they showed them in their behaviour, their strengths, current challenges, concerns, and where they'd like support. The team would perform a joint short, guided observation, bring parent and baby together, and coach parents in providing supports. Team members would then work with families to devise specific developmental care goals for their babies. The goals were recorded in the patient's chart and reviewed with bedside staff.

\section{Results/Findings}

NIDCAP Rounds were conducted 15 times in nine months with 25 families participating. Seven families participated in two sessions and 18 in one session. NIDCAP Rounds were attended by SLP (14 times), OT (14 times), neonatologist (4 times), and neonatal nurse practitioner (one time).

Developmental care goals addressed included:

- hold baby skin-to-skin (4)

- support baby to come to alertness by speaking/singing in parents' first language (8)

- support baby to fall asleep by hand swaddling and protect sleep by keeping noise, lights, and activity low (5)

- provide positioning supports to help baby maintain a comfortable flexed position in bed (4)

- nuzzle at a pumped breast whenever alert and rooting (3)

- implement supportive feeding strategies (swaddle, elevated side-lying position, gentle burping, pacing, hold upright during tube feeds) in order to support positive oral experiences (8)

- schedule interventions when parents available to provide support (3)

- gradually reduce rolls and start day/night routine in preparation for transition home (9)

\section{Relevance to NIDCAP}

This project addresses the NIDCAP Nursery Program categories of the nursery environment and care of the infant, family, and professional healthcare team.

\section{Conclusion}

By implementing NIDCAP rounds, we were able to use a small time-commitment to meet with many families.

NIDCAP observations led to parents and staff partnering to define infants individualized developmental goals.

\section{NIDCAP Trainers Meeting Feedback}

"It was rewarding to see the work NIDCAP is achieving." 